ARTÍCULO DE INVESTIGACIÓN

\title{
Ecuaciones de aditividad para estimar componentes de biomasa de Hevea brasiliensis Muell. Arg., en Veracruz, México
}

\author{
Carlos Monroy Rivera ${ }^{1}$ \\ José de Jesús Návar Chaidez ${ }^{1}$
}

\begin{abstract}
RESUMEN
La importancia de las estimaciones de volúmenes de biomasa para el manejo de recursos maderables y no maderables ha sido reconocida por las autoridades del país en años recientes. Esto se reconoce parcialmente por la implantación de programas para el pago de los servicios ambientales por parte de la Comisión Nacional Forestal. El presente trabajo se realizó en los Municipios de Tezonapa y Uxpanapa, Veracruz, México, con el objetivo de estimar coeficientes estadísticos con la menor varianza a través de la comparación de diferentes técnicas aditivas de estimación de componentes de biomasa (fuste, ramas y total) para el clon de hule IAN-710 provenientes de plantaciones comerciales para la producción de látex. Los datos de campo registrados fueron los pesos verde y seco de las ramas y fuste. Se estimaron los coeficientes de dos ecuaciones de biomasa: 1) la ecuación de la variable combinada de Spurr (1952) y, 2) el mejor modelo. Se probaron cuatro formas de estimación de parámetros: 1) regresión lineal convencional, 2) regresión lineal ponderada, 3) regresión lineal generalizada y 4) regresión lineal generalizada ponderada. Se encontró, al comparar los valores de $t$ de los parámetros, que los coeficientes con menor varianza se estimaron en regresión lineal generalizada ponderada y los estadísticos de ajuste de los modelos no cambiaron de forma significativa. Se recomienda usar la regresión desarrollada en procedimientos de regresión lineal generalizada ponderada en la estimación de componentes de biomasa para el clon de hule IAN-710.
\end{abstract}

PALABRAS CLAVE:

Biomasa del fuste, ramas y total, coeficientes con menor varianza, regresión lineal generalizada ponderada.

\begin{abstract}
The importance of estimating biomass volumes for the management of wood and non wood forest products has been recently recognized by the government of Mexico. This is partially recognized by the implementation of government programs for the payment of environmental services, by the Mexican Forestry Commission. This research was carried out at the Municipalities of Tezonapa and Uxpanapa, Veracruz, Mexico with the objective of estimating statistical coefficients with the least variance by comparing different additive techniques of biomass component estimation (stem, branch, and total) for the latex clon IAN-710 coming from commercial forest plantations for latex production. Field data collected were fresh and dry weight values. Coefficients of two biomass equations were evaluated: 1) the combined variable of Spurr (1952) and 2) the best regression model. Four ways of estimating parameters were tested: 1) conventional linear regression, 2) weighted linear regression, 3) seemingly unrelated linear regression, and 4) weighted seemingly unrelated linear regression. When comparing the $t$ values of the coefficients, it was found that the least variance coefficients were estimated in weighted seemingly unrelated linear regression and the coefficients did not show significant change. Therefore, this equation is recommended for estimating coefficients of equations for biomass components and total for the latex clon IAN-710.
\end{abstract}

KEY WORDS:

Biomass components and total, least variance coefficients, weighted seemingly linear regression. 


\section{INTRODUCCIÓN}

La estimación del volumen de la biomasa aérea de los árboles es un aspecto esencial para estudios de almacenes y flujos de carbono en los ecosistemas forestales. Por ello es necesario entender el papel que juegan las prácticas de aforestación y reforestación y deforestación en el balance global de carbono. El método destructivo es el más preciso para estimar el volumen de biomasa aérea; hay otros métodos de medición basados, por ejemplo, en el diámetro normalizado y la altura. Las ecuaciones alométricas se derivan de estos datos para estimar biomasa total y se usan además para realizar inventarios forestales y estudios ecológicos (Whittaker y Marks (1975) y Pardé (1980). Aplicaciones recientes y estimaciones de biomasa en estudios ambientales incluyen diferentes autores como: Uhl et al. (1988); Brown et al. (1989); Brown y Lugo (1992); Brown (1997); Schroeder et al. (1997); Ayala (2001) y Jaramillo et al. (2003).

La información de biomasa se ha usado para diferentes propósitos como: a) descripción cuantitativa de ecosistemas y fuentes de biomasa disponibles (Young y Tryon, 1978; Brown, 1997); b) cuantificación de la cantidad de nutrimentos en el ecosistema (Long y Turner, 1974; Golley, 1975; Baker et al., 1984; Lim, 1988); c) determinación de la fijación de energía en ecosistemas forestales (Satto, 1968); d) estimación del contenido de carbono en el bosque (Brown y Lugo, 1984; Brown et al., 1989; Brown, 1997); e) cuantificación del incremento y rendimiento de bosques y crecimiento-productividad (Burkhart y Strub, 1973) y f) evaluar cambios en la estructura del bosque (Brown, 1997).

El interés en usar el árbol completo en la manufactura de diferentes productos forestales con vida media en el ecosistema incrementa la precisión en el balance de las plantaciones de hule. Por esta razón, la estimación de los componentes de biomasa es una tarea importante dentro de los estudios ambientales. El árbol, normalmente es separado en tres componentes: a) fuste o tronco principal, b) corteza del fuste y c) copa (ramas y follaje). En ocasiones es usado un cuarto componente que incluye el tocón y raíces principales (Karizumi, 1977; Satto y Sassa, 1979 citado por Parresol, 1999; Deans et al., 1996; Kurz et al., 1996 y Reed et al., 1996). Comúnmente se desarrollan ecuaciones alométricas para cada componente del árbol por separado. Las ecuaciones alométricas simples tienen la forma $Y=a(d n)^{b}$ (Satto y Madgwick, 1982). Varias de estas ecuaciones se han desarrollado para plantaciones de especies tropicales. Se han desarrollado ecuaciones alométricas para estimar biomasa aérea de diferentes especies tropicales. Entre las ecuaciones alométricas para este tipo de especies están: Tectona grandis (Pérez y Kanninen, 2003), Eucalyptus sp. (Senelwa y Sims, 1998) y Pinus pinaster (Ritson y Sochacki, 2003). Algunos otros estudios recientes han mostrado un considerable rango en ecuaciones alométricas para bosques primarios y secundarios en los trópicos húmedos de Brasil (Alves et al., 1997; Araújo et al., 1999; Nelson et al., 1999). Para plantaciones de Eucalyptus, distintas especies del bosque subtropical lluvioso y coníferas exóticas de Nueva Gales del Sur, Australia (Spetch y West, 2003), así como para rodales de 1 año a 6 años de Gmelina arborea (Robx) de la India Central (Swamy et al., 2004).

Una característica deseable de las ecuaciones alométricas de regresión de los componentes del árbol es que la suma de las predicciones para los componentes sea igual a la predicción para el total de los árboles (Kozak, 1970; Chiyenda y 
Kozak, 1984; Cunia y Briggs, 1984, Reed y Green, 1985; Parresol, 1999; Méndez, 2001; Contreras y Návar, en rev; Návar et al., 2004a). Se ha discutido el problema de forzar la aditividad de las funciones de biomasa, resultando en tres procedimientos de acuerdo con el orden en que se incluyen los componentes individuales: 1) suma de estimadores de las mejores funciones de regresión de cada componente 2) uso de las mismas variables independientes para la estimación de cada componente y 3) forzando los coeficientes de regresión para estimar el componente total, con regresiones lineales generalizadas (SUR por sus siglas en inglés, Seemingly Unrelated Regressions) (Parresol, 1999; Návar et al., 2002). Estos procedimientos de reciente aplicación en la evaluación cuantitativa de ecosistemas templados (Cunnia y Briggs, 1984; Rose y Linch, 2001; Brooks et al., 2002; Návar et al., 2004b, en rev.) y semiáridos (Návar et al., 2002; Návar et al., 2004a; Méndez, 2001) requieren ser probados en árboles y arbustos de otros ecosistemas terrestres.

A pesar de las ecuaciones alométricas desarrolladas para una variedad de especies tropicales, se han realizado pocos intentos para generar ecuaciones que describan simultáneamente los componentes de los árboles, cuando existen ecuaciones aditivas para describir componentes de biomasa. Existe un ejemplo recientemente publicado en la literatura científica para especies mexicanas (Navár et al., 2004a) pero no se han aplicado las técnicas de aditividad para especies tropicales mexicanas y no existen muchas ecuaciones de biomasa para árboles de hule. Estas justificaciones motivaron el establecimiento del presente estudio.

\section{OBJETIVOS}

Desarrollar ecuaciones que describan de forma simultánea los componentes de biomasa, fuste, ramas y total en el clon IAN-710 de árboles de hule (Hevea brasiliensis Muell., Arg.), de los Municipios de Tezonapa y Uxpanapa, Veracruz, con la aplicación de diferentes técnicas de ajuste de parámetros.

\section{Área de estudio}

El estudio se realizó en dos localidades del estado de Veracruz. La primera corresponde a plantaciones comerciales del clon de hule IAN-710, ubicadas en el Valle de Tezonapa, Ver., en terrenos del Campo El Palmar del Instituto Nacional de Investigaciones Forestales, Agrícolas y Pecuarias (SAGARPA), ubicado en el km 16 carret. Tezonapa-El Palmar Grande, Mpio. de Tezonapa, con una altitud de $180 \mathrm{msnm}$. La segunda localidad se encuentra en el Ejido Monterrey, Municipio de Uxpanapa, Ver., a una altitud de $120 \mathrm{msnm}$. En la primera localidad el clima es cálido húmedo, con precipitación de $2885 \mathrm{~mm}$ anuales. La temperatura media es de $24,4{ }^{\circ} \mathrm{C}$ con $16,1^{\circ} \mathrm{C}$ y 35,45 ○C como mínima y máxima, respectivamente. La fisiografía es de terrenos planos y lomeríos con pendientes de $5 \%$ a $29 \%$. Los suelos son acrisoles y vertisoles, profundos y con buen drenaje natural con textura migajón arcilloarenosa y con $\mathrm{pH}$ de 4,8 a 6,1. En la segunda localidad el clima es cálido húmedo, con precipitación de $3800 \mathrm{~mm}$ anuales. La temperatura media anual es de $24^{\circ} \mathrm{C}$ con $18^{\circ} \mathrm{C}$ y $28^{\circ} \mathrm{C}$ como mínima y máxima respectivamente. Los suelos son acrisoles órticos, profundos con textura arcillosa y con $\mathrm{pH}$ de 4,3 a 5,5. 


\section{METODOLOGÍA}

\section{Muestreo y selección de árboles}

Se derribaron 3 árboles por clase de edad, seleccionados al azar, de cada una de las plantaciones de las dos localidades, existentes en tres parcelas de evaluación de $600 \mathrm{~m}^{2}(30 \mathrm{~m} \times 20 \mathrm{~m})$. La localidad de Tezonapa incluyó las edades 8 años (con únicamente dos árboles), 14 y 31 años. La localidad de Uxpanapa incluyó las edades de 14, 16, 19 y 23 años. Las edades corresponden a las plantaciones disponibles para el clon 710 de hule sujeto a estudio.

A cada árbol se le midieron y registraron las variables diámetro al nivel del suelo (DB); diámetro normal (D1,3m), altura del fuste limpio (HF); altura total (HT) y cobertura (CB). Se registró el peso húmedo y seco de cada componente de biomasa, expresado en: fustes y ramas de cada árbol muestra. El volumen de cada troza se determinó usando la fórmula de Smalian, con mediciones de diámetro en ambas secciones desde la base, a 1,30 m y a cada 2,50 m., hasta la parte distal del fuste limpio. El volumen de la punta se estimó con la fórmula del cono (Clutter et al.,1983). Se registró el peso húmedo y seco de cada componente de biomasa, expresado en fustes y ramas de cada árbol muestra. Las muestras de madera y ramas se secaron al horno por 24 horas a $65^{\circ} \mathrm{C}$.

\section{Especie seleccionada}

La especie sujeta a estudio corresponde al clon de hule (Hevea brasiliensis Muell. Arg.) IAN-710, establecido en plantaciones como monocultivo en áreas sensiblemente planas del trópico húmedo de México, particularmente en rodales de los Valles de Tezonapa y Uxpanapa, Ver. Únicamente en estas localidades se encuentran individuos del clon de hule IAN-710 con diámetros apropiados para el cálculo de las ecuaciones de biomasa, aunque otras localidades como Las Choapas, Ver. y Palenque, Chis., presentan plantaciones muy jóvenes (5 años a 10 años) del clon de hule en estudio. Se colectó una muestra de dos localidades diferentes y los parámetros dasométricos $(d, h)$ son diferentes para árboles de la misma edad. Las existencias reales del fuste varían de $52 \mathrm{~m}^{3}$ ha-1 a $162 \mathrm{~m}^{3} \mathrm{ha}^{-1}$ (Gan et al., 1985). En consecuencia el volumen de madera por hectárea depende de numerosos factores tales como clon, sitio, manejo, edad, etc. Algunos pequeños productores señalan que los troncos del árbol de hule son de baja calidad, atribuido a la falta de manejo silvícola, así como por el efecto de incisión en el tablero de pica o sangrado que afectan las propiedades de la madera. En un estudio realizado en Johore, Malasia, se registró que sólo el $18 \%$ de troncos de madera de hule cosechados en áreas de pequeños productores puede ser usado para madera aserrada (Norini y Zana, 1993 citado por Killman y Hong, 2000).

\section{Procedimiento estadístico}

En esta investigación se utilizaron cuatro tipos de procedimientos para estimar coeficientes de dos tipos de ecuaciones alométricas para los componentes de biomasa. Los datos de componentes de peso seco de fuste, peso seco de ramas y peso seco total (fuste + ramas); además de los datos de diámetro normal, $\mathrm{D}$, y altura total, $\mathrm{H}$, de un conjunto de 20 árboles fueron usados para el ajuste de las ecuaciones de biomasa estimadas. Se utilizó el ajuste de dos tipos de modelos: 1) una sola variable y 2) el mejor modelo. En el primer caso se uso la variable combinada de Spurr (1952) $B_{i}=b_{0}+$ $b_{1} D^{2} H$, por ser una ecuación que presentó los mejores estadísticos de bondad de ajuste $\left(r^{2}\right.$, Sx y CV), de un conjunto de 
ecuaciones lineales de biomasa ensayadas con Paquete estadístico SAS (versión 8.1). En el segundo caso, los mejores modelos de biomasa se seleccionaron con procedimientos STEPWISE. Las variables usadas fueron: $D, D^{2}, D^{2} H, D H^{2}$ y $D^{2} H F$. La selección del mejor modelo resulta en alguna de las ecuaciones alométricas probadas anteriormente (Clutter et al., 1983; Wenger, 1984; Bailey, 1994; Prodan et al., 1997).

Los coeficientes de cada uno de los dos tipos de modelos se calcularon (SAS, versión 8.1) de cuatro formas diferentes: 1) regresión lineal convencional sin ponderar, 2) regresión lineal ponderada, 3) regresión lineal generalizada y 4) regresión lineal generalizada ponderada. El uso de la ponderación se realizó con el fin de modelar la estructura del error y eliminar o reducir el problema de heterocedasticidad, con base en que el error se puede predecir y utilizar como ponderador.

La selección de la mejor técnica para estimar los coeficientes estadísticos resultó de comparar: 1) los estadísticos de ajuste y 2) valores de $t$ de los parámetros y su respectivo nivel de probabilidad. Los estadísticos de bondad de ajuste usados fueron: 1) el coeficiente de determinación, $r^{2}$, 2) el error estándar, Sx; y 3) el coeficiente de variación de los residuales, CV. Los valores de $t$ de los parámetros resultan de la división del estimador entre su error estándar. La significancia estadística del valor $t$ está dada por el cálculo de la probabilidad. Este es un procedimiento recomendado por Návar et al. (2004b, en rev.) para el desarrollo de ecuaciones de biomasa.

\section{RESULTADOS Y DISCUSIÓN}

Las características dasométricas promedio de las variables primarias del clon de hule IAN-710 en el centro y sur de
Veracruz de donde se extrajeron las muestras para el cálculo de las ecuaciones de biomasa se presentan en la Tabla 1.

De manera análoga los estadísticos de las variables dasométricas y biomasa de 20 árboles evaluados del clon de hule (Hevea brasiliensis Muell. Arg. ) IAN-710 se señalan en la Tabla 2.

\section{Componentes de biomasa}

El promedio de los componentes de la biomasa medida para el fuste y ramas fue $73,88 \%$ y $27,09 \%$, respectivamente. Los componentes de biomasa variaron con la edad de plantación. En las edades de $31,23,19,16,14$ y 8 años, la biomasa medida en el componente fuste fue 80,03 $\%, 64,24 \%, 81,84 \%, 65,55 \%, 79,63 \%$ y $65,71 \%$, respectivamente. Asimismo, para las mismas edades, la biomasa del componente ramas medida fue $19,96 \%$, $35,75 \%, 18,15 \%, 34,44 \%, 20,36 \%$ y $34,28 \%$, respectivamente. El contenido más alto de biomasa en fuste se registró para la edad de 31 años, mientras que la biomasa más alta de ramas se registró para la edad de 23 años.

Los parámetros de la ecuación generalizada de Spurr (1952) y los estadísticos de bondad de ajuste se muestran en la Tabla 3. Los diferentes procedimientos de estimación de parámetros resultaron en diferentes coeficientes. Los cambios más notorios resultaron cuando se ponderaron las ecuaciones en contraste a cuando se desarrollaron en procedimientos sin ponderar. Por esta razón, los parámetros estadísticos de ajuste de las ecuaciones cambian aunque lo hacen de una forma no muy notable. En particular, la regresión simple sin ponderar sería la más adecuada para estimar componentes de biomasa y total de Hevea brasiliensis Muell. Arg. La disminución en los estadísticos de ajuste 
es el precio que se paga en la estimación de los parámetros con procedimientos de regresión lineal generalizada, al forzar la suma de los coeficientes a resultar en los coeficientes de la ecuación de la biomasa total. Para la regresión ponderada, también en general, disminuye ligeramente el valor de $r^{2}$ y aumentan ligeramente los valores de Sx y CV, pero incre- menta la precisión de los parámetros de la ecuación (Návar et al., 2004b).

Los parámetros y ecuaciones resultantes del procedimiento STEPWISE y los estadísticos de bondad de ajuste para estimar los componentes de biomasa se presentan en la Tabla 4.

Tabla 1. Características dasométricas promedio de los árboles del clon IAN-710 de centro y sur de Veracruz de donde se extrajeron los individuos para medir los componentes de biomasa y estimar las ecuaciones

\begin{tabular}{|c|c|c|c|c|c|c|}
\hline LOCALIDAD & $\begin{array}{l}\text { EDAD } \\
\text { (años) }\end{array}$ & $\begin{array}{l}\mathrm{DB} \\
(\mathrm{cm})\end{array}$ & $\mathrm{D}_{1,3 \mathrm{~m}}$ & $\mathrm{H}(\mathrm{m})$ & $\begin{array}{l}\mathrm{HF} \\
(\mathrm{m})\end{array}$ & $\begin{array}{l}\mathrm{CB} \\
\left(\mathrm{m}^{2}\right)\end{array}$ \\
\hline \multirow{3}{*}{ Tezonapa } & 8 & 27,0 & 24,5 & 15,7 & 7,90 & 30,72 \\
\hline & 14 & 30,6 & 28,5 & 20,5 & 11,70 & 21,53 \\
\hline & 31 & 51,0 & 47,0 & 26,7 & 14,80 & 59,60 \\
\hline \multirow{4}{*}{ Uxpanapa } & 14 & 26,0 & 22,3 & 17,60 & 9,90 & 9,30 \\
\hline & 16 & 29,0 & 25,6 & 20,40 & 11,30 & 10,89 \\
\hline & 19 & 29,6 & 26,3 & 24,9 & 14,10 & 9,96 \\
\hline & 23 & 41,3 & 38,0 & 26,6 & 16,60 & 53,87 \\
\hline $\begin{array}{l}\mathrm{DB}=0 \\
\mathrm{D}_{1,3 \mathrm{~m}}=0\end{array}$ & $\begin{array}{l}\text { tro basal } \\
\text { tro normal }\end{array}$ & $\begin{array}{l}\mathrm{H} \\
\mathrm{HF}\end{array}$ & $\begin{array}{l}\text { total } \\
\text { fuste }\end{array}$ & CB & & \\
\hline
\end{tabular}

Tabla 2. Estadísticos de la variables dasométricas y biomasa de 20 árboles del clon de hule (Hevea brasiliensis Muell. Arg.) IAN-710 en Veracruz, México

\begin{tabular}{|c|c|c|c|c|c|c|}
\hline \multirow[t]{2}{*}{ PARÁMETRO } & \multicolumn{3}{|c|}{$\begin{array}{c}\text { VARIABLES } \\
\text { DASOMÉTRICAS }\end{array}$} & \multicolumn{3}{|c|}{ BIOMASA PROMEDIO } \\
\hline & $\begin{array}{l}\text { Diámetro } \\
\quad(\mathrm{cm})\end{array}$ & $\begin{array}{l}\text { Altura } \\
(\mathrm{m})\end{array}$ & $\begin{array}{l}\text { Edad } \\
\text { (años) }\end{array}$ & $\begin{array}{l}\text { Fuste } \\
(\mathrm{kg})\end{array}$ & $\begin{array}{l}\text { Ramas } \\
\text { (kg) }\end{array}$ & $\begin{array}{l}\text { Total } \\
(\mathrm{kg})\end{array}$ \\
\hline Promedio & 31 & 22,0 & 18,3 & 374 & 137,2 & 506,3 \\
\hline $\begin{array}{l}\text { Desv. } \\
\text { Estándar }\end{array}$ & 9,2 & 4,08 & 6,84 & 284 & 119,2 & 363,0 \\
\hline $\begin{array}{l}\text { Intervalo } \\
\text { Confianza }\end{array}$ & 4,1 & 1,82 & 3,08 & 127 & 53,3 & 162,3 \\
\hline
\end{tabular}


Tabla 3. Parámetros de ecuaciones de biomasa de la ecuación generalizada de Spurr (1952) y estadísticos de bondad de ajuste para estimar componentes de biomasa de 20 árboles de Hevea brasiliensis Muell. Arg., en Veracruz, México

\begin{tabular}{|c|c|c|c|}
\hline \multirow[t]{2}{*}{ PARÁMETROS } & \multicolumn{3}{|c|}{$\begin{array}{c}\text { ESTADÍSTICOS DE BONDAD } \\
\text { DE AJUSTE }\end{array}$} \\
\hline & $r^{2}$ & Sx & $\mathrm{CV}$ \\
\hline \multicolumn{4}{|l|}{ ECUACIÓN SIN PONDERAR } \\
\hline$B_{\text {fuste }}=37,35705+0,013422 D^{2} H$ & 0,85 & 114,4 & 0,30 \\
\hline$B_{\text {ramas }}=29,99596+0,004273 D^{2} H$ & 0,49 & 89,72 & 0,65 \\
\hline$B_{\text {total }}=67,35301+0,017695 D^{2} H$ & 0,84 & 158,4 & 0,30 \\
\hline \multicolumn{4}{|l|}{ ECUACIÓN PONDERADA } \\
\hline$B_{\text {fuste }}=10,3823+0,014562 D^{2} H$ & 0,84 & 116,8 & 0,31 \\
\hline$B_{\text {ramas }}=0,729187+0,005356 D^{2} H$ & 0,46 & 92,5 & 0,67 \\
\hline$B_{\text {total }}=35,80424+0,01897 D^{2} H$ & 0,83 & 160,64 & 0,31 \\
\hline \multicolumn{4}{|l|}{ ECUACIÓN LINEAL GENERALIZADA } \\
\hline$B_{\text {fuste }}=37,35705+0,013422 D^{2} H$ & 0,85 & 114,4 & 0,30 \\
\hline$B_{\text {ramas }}=29,99596+0,004273 D^{2} H$ & 0,49 & 89,7 & 0,65 \\
\hline$B_{\text {total }}=67,35301+0,017695 D^{2} H$ & 0,70 & 215,9 & 0,42 \\
\hline \multicolumn{4}{|c|}{ ECUACIÓN LINEAL GENERALIZADA PONDERADA } \\
\hline$B_{\text {fuste }}=16,02547+0,013939 D^{2} H$ & 0,85 & 115,3 & 0,30 \\
\hline$B_{\text {ramas }}=-2,52112+0,005151 D^{2} H$ & 0,46 & 92,24 & 0,67 \\
\hline$B_{\text {total }}=13,50436+0,019091 D^{2} H$ & 0,83 & 162,3 & 0,31 \\
\hline
\end{tabular}

Se observa que los parámetros de las ecuaciones cambian entre procedimientos de estimación. Asimismo los parámetros de la ecuación desarrollada en procedimientos de regresión lineal generalizada sin ponderar, resultan en valores intermedios al compararse con los valores de los procedimientos convencionales con y sin ponderar.

Los coeficientes de la regresión lineal generalizada ponderada presen- taron desviaciones notables en los valores de los coeficientes cuando se compararon con los obtenidos con los otros procedimientos. Aún cuando los coeficientes de los modelos se desviaron entre procedimientos, los estadísticos de bondad de ajuste $r^{2}$, Sx, y CV, no registraron cambios significativos entre los cuatro procedimientos de ajuste de coeficientes. 
Tabla 4. Parámetros y ecuaciones resultantes del procedimiento STEPWISE y estadísticos de bondad de ajuste para estimar componentes de biomasa de 20 árboles de

Hevea brasiliensis Muell. Arg., en Veracruz, México

\begin{tabular}{|c|c|c|c|}
\hline \multirow[t]{2}{*}{ PARÁMETROS } & \multicolumn{3}{|c|}{$\begin{array}{l}\text { ESTADÍSTICOS DE } \\
\text { BONDAD DE AJUSTE }\end{array}$} \\
\hline & $r^{2}$ & Sx & CV \\
\hline \multicolumn{4}{|l|}{ ECUACIÓN SIN PONDERAR } \\
\hline$B_{\text {fuste }}=-277,407+0,010377 D^{2} H+17,75782 H$ & 0,87 & 105,7 & 0,28 \\
\hline$B_{\text {ramas }}=-146,797+9,124540 D$ & 0,49 & 86,57 & 0,63 \\
\hline$B_{\text {totd }}=-517,289+0,010121 D^{2} H+25,45754 H+6,872439 D$ & 0,86 & 150,24 & 0,29 \\
\hline \multicolumn{4}{|l|}{ ECUACIÓN PONDERADA } \\
\hline$B_{\text {fuste }}=-377,321+0,009784 D^{2} H+23,17905 H$ & 0,87 & 106,9 & 0,28 \\
\hline$B_{\text {ramas }}=-146,855+9,086030 \mathrm{D}$ & 0,49 & 89,09 & 0,64 \\
\hline$B_{\text {total }}=-447,042+0,013448 D^{2} H+28,23999 H+0,146205 D(3)$ & 0,86 & 147,0 & 0,28 \\
\hline \multicolumn{4}{|l|}{ ECUACIÓN LINEAL GENERALIZADA } \\
\hline$B_{\text {fuste }}=-256,606+0,010576 D^{2} H+16,58630 H$ & 0,87 & 105,78 & 0,28 \\
\hline$B_{\text {ramas }}=-146,999+9,131030 D$ & 0,49 & 89,08 & 0,64 \\
\hline$B_{\text {total }}=403,605+0,010576 D^{2} H+16,58630 H+9,131030 D(3)$ & 0,86 & 147,4 & 0,28 \\
\hline \multicolumn{4}{|l|}{ ECUACIÓN LINEAL GENERALIZADA PONDERADA } \\
\hline$B_{\text {fuste }}=-442,174+0,008684 D^{2} H+27,48022 H$ & 0,86 & 108,6 & 0,29 \\
\hline$B_{\text {ramas }}=-106,273+6,948465 D$ & 0,41 & 96,21 & 0,70 \\
\hline$B_{\text {total }}=-548,448+0,008684 D^{2} H+27,48022 H+6,948465 D(3)$ & 0,86 & 149,0 & 0,29 \\
\hline
\end{tabular}

Bfuste $=$ biomasa fuste $\quad$ Bramas $=$ biomasa ramas

Btotal $=$ biomasa total

Los valores de $t$ de los parámetros de las ecuaciones para los componentes de biomasa: fuste, ramas y total, muestran que la regresión lineal generalizada ponderada presenta los valores de $t$ mayores. En general, los valores de $t$ incrementaron en $20 \%$, $30 \%$ y $5 \%$ en los coeficientes estimados con regresión lineal generalizada ponderada en contraste con regresión lineal simple, regresión lineal simple ponderada y en regresión lineal generalizada, respectivamente. Esto es indicativo del incremento en la precisión de los parámetros. Es decir, los parámetros estimados en regresión lineal generalizada ponderada contienen una menor varianza y los hace más deseables como estimadores de los coeficientes de regresión.

Asimismo los valores de $t$ y nivel de probabilidad de los coeficientes de cuatro formas de estimación de parámetros del clon de hule IAN-710 se muestran en la Tabla 5. 
Tabla 5. Valores de $t$ y nivel de probabilidad de los coeficientes estadísticos de cuatro formas de estimación de parámetros de ecuaciones de biomasa del clon de hule IAN-710 en Veracruz

\begin{tabular}{|c|c|c|c|c|c|c|}
\hline \multirow[t]{2}{*}{ COMPONENTE } & \multicolumn{2}{|c|}{ UNA VARIABLE } & \multicolumn{4}{|c|}{ MEJOR MODELO } \\
\hline & a & $\mathrm{D}^{2} \mathrm{H}$ & a & $D^{2} H$ & $\mathrm{H}$ & $\mathrm{D}$ \\
\hline \multicolumn{7}{|c|}{ ECUACIÓN SIN PONDERAR } \\
\hline B fuste: (1) & $\begin{array}{c}0,91 \\
\text { n.s. }\end{array}$ & $\underset{* *}{10,2}$ & $\underset{*}{1,47}$ & $\underset{* \star}{4,78}$ & $\underset{\star \star}{1,71}$ & \\
\hline Bramas : (2) & $\begin{array}{l}0,93 \\
\text { n.s. }\end{array}$ & $\underset{\star \star *}{4,18}$ & 2,11 & & & $\underset{\star \star *}{4,24}$ \\
\hline $\mathrm{B}_{\text {total }}:(3)$ & $\begin{array}{l}1,18 \\
\text { n.s. }\end{array}$ & $\underset{* *}{9,8}$ & $\begin{array}{c}0,84 \\
\text { n.s. }\end{array}$ & $\begin{array}{c}0,77 \\
\text { n.s. }\end{array}$ & $\underset{*}{1,71}$ & $\begin{array}{c}0,26 \\
\text { n.s. }\end{array}$ \\
\hline \multicolumn{7}{|c|}{ ECUACIÓN PONDERADA } \\
\hline$B_{\text {fuste: }}$ (1) & $\begin{array}{l}0,30 \\
\text { n.s. }\end{array}$ & $\underset{* *}{8,09}$ & $\begin{array}{l}4,4 \\
\star \star\end{array}$ & $\underset{* \star}{5,17}$ & $\underset{* \star}{4,25}$ & \\
\hline Bramas: (2) & $\begin{array}{l}0,04 \\
\text { n.s. }\end{array}$ & $\underset{\star \star}{4,17}$ & $\underset{\star \star}{2,17}$ & & & $\underset{* *}{3,55}$ \\
\hline$B_{\text {total }}:(3)$ & $\begin{array}{c}0,72 \\
\text { n.s. }\end{array}$ & $\underset{\star *}{8,43}$ & $\begin{array}{l}1,53 \\
\text { n.s. }\end{array}$ & $\begin{array}{c}1,58 \\
\text { n.s. }\end{array}$ & $\underset{\star \star}{3,19}$ & $\begin{array}{c}0,01 \\
\text { n.s. }\end{array}$ \\
\hline \multicolumn{7}{|c|}{ ECUACIÓN LINEAL GENERALIZADA (SUR SIN PONDERAR) } \\
\hline$B_{\text {fuste: }}$ (1) & $\begin{array}{c}0,91 \\
\text { n.s. }\end{array}$ & $\underset{* *}{10,2}$ & $\begin{array}{l}1,4 \\
\text { n.s. }\end{array}$ & $\underset{\star \star}{4,99}$ & $\underset{*}{1,64}$ & \\
\hline Bramas: (2) & $\begin{array}{c}0,93 \\
\text { n.s. }\end{array}$ & $\underset{* \star}{4,18}$ & $\underset{* \star}{2,11}$ & & & $\underset{\star *}{3,79}$ \\
\hline $\mathrm{B}_{\text {total }}$ : (3) & $\begin{array}{l}1,18 \\
\text { n.s. }\end{array}$ & $\underset{* *}{9,8}$ & $\underset{* \star}{2,04}$ & $\underset{* *}{4,99}$ & $\underset{*}{1,64}$ & $\underset{\star \star *}{4,25}$ \\
\hline \multicolumn{7}{|c|}{ ECUACIÓN LINEAL GENERALIZADA PONDERADA (SUR PONDERADA) } \\
\hline $\mathrm{B}_{\text {fuste }}:(1)$ & $\begin{array}{l}0,53 \\
\text { n.s. }\end{array}$ & $\underset{\star \star}{9,48}$ & $\underset{\star \star}{6,02}$ & $\underset{\star \star}{5,38}$ & $\underset{\star \star}{5,91}$ & \\
\hline Bramas: (2) & $\begin{array}{l}0,23 \\
\text { n.s. }\end{array}$ & $\underset{\star \star \star}{5,1}$ & $\underset{*}{2,29}$ & & & $\underset{\star \star}{3,79}$ \\
\hline $\mathrm{B}_{\text {total }}:(3)$ & $\begin{array}{l}0,47 \\
\text { n.s. }\end{array}$ & $\underset{* *}{12,0}$ & $\underset{* *}{6,36}$ & $\underset{* *}{5,38}$ & $\underset{\star *}{5,91}$ & $\underset{* *}{3,79}$ \\
\hline attamente sic & & nas & & $\begin{array}{l}\text { Btota } \\
\text { n.s. }\end{array}$ & dse & \\
\hline
\end{tabular}


Los mayores valores de $t$ resultan principalmente de la reducción del error estándar de los parámetros. Debido a esto, el procedimiento SUR Ponderado registró los coeficientes estadísticos más precisos o con menor varianza y por consiguiente es el más eficiente, en particular para el modelo de estimación de la biomasa total, cuando se usa el criterio de la variable combinada.

En general los modelos aditivos para el cálculo de la biomasa total se caracterizan por registrar todos los coeficientes con significancia estadística notable. El modelo aditivo SUR sin ponderar registró altos coeficientes estadísticos en comparación con los estimados por los procedimientos convencionales.

Los resultados de las observaciones de los valores de $t$ y sus probabilidades confirman que los procedimientos SUR aumentan la precisión de los parámetros al utilizar de manera simultánea el número de árboles por el número de compartimentos en la estimación de la varianza total. Para el caso de este estudio, el cálculo de las ecuaciones fue sobre 60 datos (datos de fuste + datos de ramas + datos de la biomasa total). Este es un procedimiento estadístico de carácter novedoso y no aplicado hasta ahora en la mayoría de las especies tropicales como el género Hevea.

Así mismo el proceso de ponderación aumenta la confiabilidad en la estimación de parámetros cuando se compara con el procedimiento convencional. También mejoran la precisión en la estimación de parámetros al analizar la estructura del error, al contribuir a minimizar el comportamiento de la varianza heterogénea o heterocedasticidad, una característica implícita de las variables dasométricas (Bailey, 1994) que estiman la biomasa o volumetría de recursos forestales.
Es notorio que la ecuación para ramas parece mostrar un sesgo por su comportamiento lineal, en contraste con las ecuaciones cuadráticas usadas generalmente en la literatura científica. Es decir, la biomasa de ramas crece linealmente con el incremento en diámetro, cuando en la literatura esta ecuación generalmente tiene la forma de potencia para varios componentes de biomasa. A este respecto, es claro que esta ecuación es válida para árboles de hasta $50 \mathrm{~cm}$ de diámetro. En este estudio, la distribucion de diámetros de los árboles sujetos a muestreo no va más allá de $50 \mathrm{~cm}$ (Tabla 6) por lo que es válido aplicar la ecuación. Para árboles de mayores dimensiones, la tendencia entre la biomasa ramal observada y predicha puede incrementarse notoriamente. Las implicaciones biológicas, ecológicas y silvícolas de este comportamiento de la biomasa de ramas merecen atención adicional en futuras investigaciones.

Los coeficientes de regresión seleccionados junto con sus gráficas, en relación exclusiva con la variable diámetro se muestran en la figura 1. El traslape entre las líneas es notorio en el rango de la biomasa observada, indicando que la ecuación SUR ponderada para componentes de biomasa predicen valores similares a la ecuación convencional que predice sólo la biomasa total. Esta justificación adicional hace deseable la ecuación recomendada porque además genera los datos de los componentes individuales de biomasa. La ecuación tiene validez en el rango en diámetros desde $15 \mathrm{~cm}$ en delante. Los componentes de biomasa fustal y ramas se pueden predecir en árboles desde 17,5 $\mathrm{cm}$ en delante. Por debajo de este diámetro, las ecuaciones predicen biomasa negativa y se requiere de datos de árboles pequeños para ajustar esta técnica matemática. 
Tabla 6. Mapa de distribución de la muestra para ajustar las ecuaciones aditivas de biomasa de Hevea brasiliensis Muell. Arg., en Veracruz, México

\begin{tabular}{|c|c|c|c|c|c|c|c|}
\hline$D H$ & 5 & 10 & 15 & 20 & 25 & 30 & TOTAL \\
\hline \multicolumn{8}{|l|}{5} \\
\hline \multicolumn{8}{|l|}{10} \\
\hline \multicolumn{8}{|l|}{15} \\
\hline 20 & & & 1 & & & & 1 \\
\hline 25 & & & 2 & 6 & 2 & & 10 \\
\hline 30 & & & & 2 & 1 & & 3 \\
\hline 35 & & & & & 2 & & 2 \\
\hline \multicolumn{8}{|l|}{40} \\
\hline 45 & & & & & 1 & 1 & 2 \\
\hline 50 & & & & & 2 & & 2 \\
\hline \multicolumn{8}{|l|}{55} \\
\hline Total & & & 3 & 8 & 8 & 1 & 20 \\
\hline
\end{tabular}

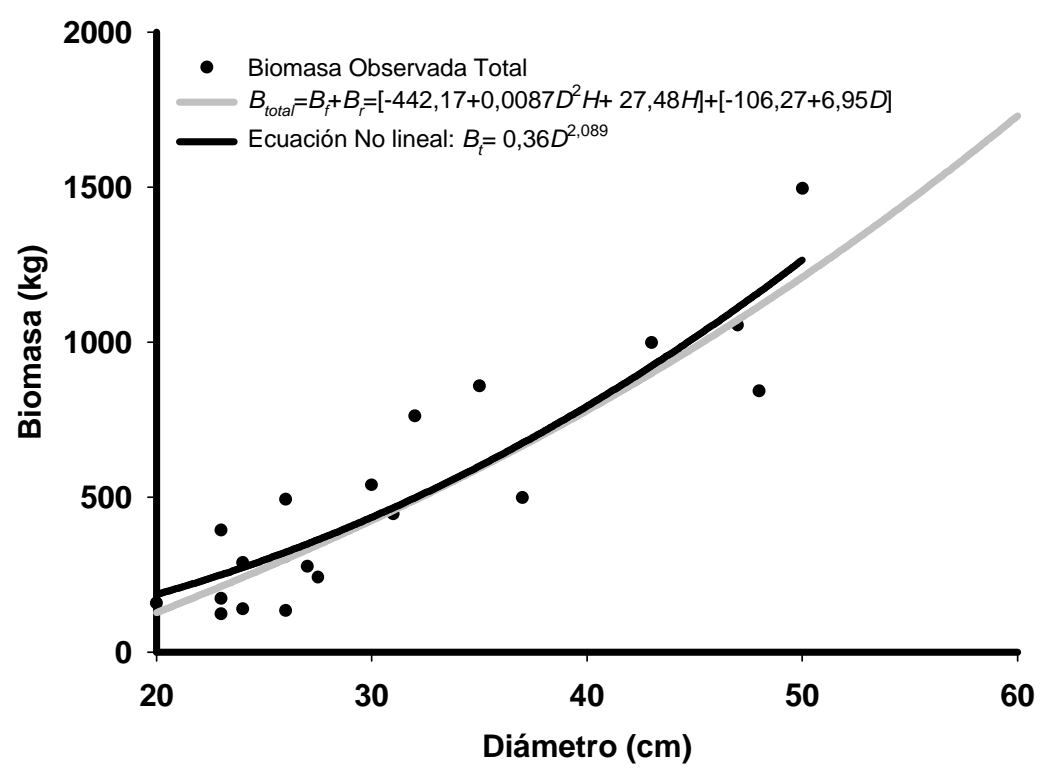

Figura 1. Comparación de ajuste de ecuaciones de componentes de biomasa SUR ponderada y convencional en Hevea brasiliensis Muell. Arg. 


\section{CONCLUSIONES}

- Los resultados de pruebas de bondad de ajuste de cuatro formas de estimación de parámetros de ecuaciones de componentes de biomasa (fuste, ramas y total) de 20 árboles del clon de hule (Hevea) IAN-710, fueron suficientes para la recomendación del procedimiento adecuado en la estimación de parámetros de las ecuaciones aditivas de componentes de biomasa y total. La ecuación tiene validez en el rango en diámetros desde $15 \mathrm{~cm}$ en delante para biomasa total y de 17,5 $\mathrm{cm}$ para componentes individuales de biomasa.

- Los parámetros estimados con el procedimiento de regresión lineal generalizada con ponderación registraron precisión suficiente, comprobado por sus valores de $t$.

- La mayor parte de los coeficientes de los modelos aditivos para estimar la biomasa total presentaron evidencia estadística altamente significativa al compararse con los procedimientos de estimación convencionales. Por esta razón se recomienda la ecuación desarrollada en regresión lineal generalizada ponderada para la estimación de los componentes de la biomasa y total del árbol del hule.

\section{REFERENCIAS}

Alves, D.S.; J.S. Soares; S. Amaral; E.M. K. Mello, S.A.S. Almeida; O.F. da Silva; A.M. Silveira. 1997. Biomass of primary and secondary vegetation in Rondonia, Western Brazilian Amazonian. Climate Change Biology 3:451-461.

Araújo, T.M.; N. Higuchi y J.A. Carvalho Junior. 1999. Comparison of formulae for biomass content deter- mination in a tropical rain forest site in the state of Para, Brazil. Forest Ecology and Management 117:43-52.

Ayala, R.A. 2001. Ecuaciones para estimar biomasa de pinos y encinos en la meseta central de Chiapas. Tesis de Licenciatura. Universidad Autónoma de Chapingo. División de Ciencias Forestales. 70 p.

Baker, T.G.; P.M. Attiwill y H.T.L. Stewart. 1984. Biomass equations for Pinus radiata in Gippslanmd, Victoria. New Zealand of Forest Science 14:89-96.

Bailey, L.R. 1994. A compatible volumetaper model based on the Schumacher and Hall generalizad constant form factor volume equation. For.Sci. Vol. 40(2):303-313.

Brooks, J.R.; S. Martin; J. Jordan; C. Sewell. 2002. Interim taper and cubic-foot volume equations for young longleaf pine plantations in Southwest Georgia Gen. Tech. Rep. SRS-48. Asheville, NC: U.S. Department of Agriculture, Forest Service. Southern Research Station. p:467-470.

Brown, S. y A.E. Lugo. 1984. Biomass of tropical forest. A new estimates based on forest volumes. Science 223:1290-1293.

Brown, S., 1997. Estimating biomass and biomass change of tropical forests. FAO Forestry paper No. 134. FAO, Roma. 55 p.

Brown, S., y A.E. Lugo.1992. Aboveground biomass estimates for tropical moist forests of the Brazilian Amazon. Interciencia 17:8-18.

Brown, S.; A.J.R. Gillespie y A.E. Lugo. 1989. Biomass estimation methods for tropical forests with applications 
to forest inventory data. For. Sci. 35: 881-902.

Burkhart, H.E., y Strub, M.R. 1973. Dry weight yield estimation for Loblolly pine: a comparison of two techniques. IUFRO Biomass studies. Univ. Maine, Crona, Maine. p:27-40.

Chiyenda, S.S. y A. Kozak. 1984. Additivity of component biomass regression equations when the underlying model is linear. Can. J. For. Res. 14:441-446.

Clutter, J.L.; J.C. Fortson; L.V. Piennar; G.H. Brister y R.L. Bailey. 1983. Timber management: A quantitative approach. John Wiley. Nueva York. $333 \mathrm{p}$.

Contreras A., J.C. y J.J. Návar C. 2004. Ecuaciones aditivas para estimar componentes de volumen para Pinus teocote Schl. et Cham. de Durango, México. 10p. En revisión Ciencia Forestal en México.

Cunia, T. y R.D. Briggs. 1984. Forcing additivity of biomass tables: some empirical results. Can. J. For. Res. 14: 376-384.

Deans, J.D.; J. Moran y J. Grace. 1996. Biomass relationships for tree species in regenerating semi-deciduous tropical moist forest in Cameroon. For. Ecol. Management 88:215-225.

Gan, L.T.; C.E. Ho, y O.K. Chew. 1985. Heveawood: sawntimber production and recovery studies. Paper presented at the International Rubber Conference. Kuala Lumpur Malaysia. Malaysia. $1 \mathrm{p}$.

Golley, F.B. 1975. Mineral cicling in a tropical moist forest ecosystems.
University of Georgia. Press Athens. $248 \mathrm{p}$.

Jaramillo, V.J.; J. Boone, L. Rentería R.; D.L. Cummins y L. Ellingson. 2003. Biomasa, carbon and nitrogen pool in Mexican tropical dry landscapes Ecosystems 6:609-629.

Karizumi, N. 1977. Methods for estimating root biomass. In: Shidei, T. y T. Kira (eds). Primary productivity of Japanese forests. Japanece IBP synthesis Series Vol. 16. Univ. of Tokyo Press. Tokio. p:25-29.

Killman, W. y L.T. Hong. 2000. El caucho, el éxito de un sub producto agrícula. Unasylva. Núm. 201. Vol. 51. 12 p.

Kozak, A. 1970. Methods of ensuring additivity of biomass components by regression analysis. For Chron. 46(5):402-404.

Kurz, W.A.; S.J. Beukema y M.J. Apps. 1996. Estimation of root biomass and dynamics for the carbon budget model of the Canadian forest sector. Can. J. For. Res. 26:1973-1979.

Lim, M.T. 1988. Studies on Acacia mangium in Kemasul, Malysia. I Biomass and productivity. Journal of Tropical Ecology 4:293-302.

Long, J.N. y J. Turner.1974. Aboveground biomass of understory and overstory in an age sequence of Douglas fir stands. Journal of Applied Ecology 12:179-187.

Méndez, G.J. 2001. Ecuaciones de biomasa para especies de matorral espinoso tamaulipeco del nordeste de México. Datos con aplicaciones para inventarios de biomasa. Tesis de Maestría. Facultad de Ciencias Forestales. UANL. México. 60 p. 
Návar C., J.J.; J. Nájera; E. Méndez y V. Dale. 2002a. Estimating stand biomass in the Tamaulipan thornscrub of northeastern México. Annals of Forest Science 59(8):813-821.

Návar C., J.J.; J. Nájera y E. Jurado. 2002b. Biomass estimation equations in the Tamaulipan thornscrub of north-eastern México. Journal of Arid Environments 52:167-179.

Návar C., J.J.; N. González B.; J.J. Graciano L.; Dale, V., y Parresol, B. 2004a. Additive biomass equations for pine species of forest plantations of Durango, Mexico. Madera $y$ Bosques 10(2):17-28.

Návar C., J.J.; E. Méndez-González; J. Graciano-Luna; V. Dale, y B. Parresol. 2004b. Biomass equations for shrub species of Tamaulipan thornscrub of northeastern Mexico. Aceptado para Publicación en Journal of Arid Environments.

Nelson, B.W.; R. Mesquita, J.LG. Pereira, S.G.A. De Souza; G.T. Batista y L.B. Couto. 1999. Allometric regressions for improved estimate of secondary forest biomass in the central Amazon. Forest Ecology and Management 117: 149-167.

Pardé, D.R. 1980. Forest biomass. For. Abst. 41:343-362.

Parresol, B. 1999. Assesing tree and stand biomass: a review with examples and critical comparisons. For. Sci. 45:573-593.

Pérez, L.D. y M. Kanninen. 2003. Aboveground biomass of Tectona grandis plantations in Costa Rica. Journal of Tropical Forest Science 15(19):199-213.
Prodan, M.; R. Peters; F. Cox y P.L. Real. 1997. Mensura Forestal. Instituto Interamericano de Cooperación para la Agricultura (IICA), Deutsche Gesellschaft fur Technische Zusammennarbeit (GTZ) GMBH. San José, Costa Rica. 586 p.

Reed, D. y E.J. Green. 1985. A method of forcing additivity of biomass tables when using nonlinear models. Can. J. For. Res. 15:1184-1187.

Reed, D.D.; H.O. Liechty; E.A. Jones y Y. Zhang. 1996. Above and belowground dry matter accumulation pattern derived from dimensional biomass relationships. For. Sci. 42:236-241.

Ritson, P. y C. Sochacki. 2003. Measurements and prediction of biomass and carbon content of Pinus pinaster trees in farm forestry plantations. South - Western Australia. Forest Ecology and Management 175:103117.

Rose Ch., E. y T.B. Linch. 2001. Estimating parameters for tree basal area growth with a system of equations and seemingly unrelated regressions. Forest Ecology and Management 148:51-61.

Satto, T. 1968. Primary production and distribution of produced dry matter in a plantation of Cinnamomum camphora. Bulletin of Tokio University Forestry. Malayan Nature Journal 41:275-290.

Satto, T. y H. Madgwick. 1982. Forest biomass. W. Junk Publishers. The Hague. Holanda. 152 p.

Schroeder, P.E.; S. Brown; J. Mo; R. Birdsey y C. Cieszewski. 1997. Biomass estimation for temperate 
broadleaf forests of the U.S. using inventory data. For. Sci. 11:258-264.

Senelwa, K. y E.H. Sims R. 1998. Fuel characteristics of short rotation forest biomass. Biomass and Bioenergy 17(2):127-140.

Specht, A. y P.W. West. 2003. Estimation of biomass and sequestration carbon on farm forest plantations in Northern New South Wales, Australia. Biomass and Bioenergy 25(4):363-379.

Spurr, S.H. 1952. Forest inventory. Ronald Press. Nueva York. 476 p.

Swamy, S.L.; S.K. Kushwaha y S. Puri. 2004. Tree growth, biomass, allometry and nutrient distribution in Gmelina arborea stands grown in red lateritic soils of Central India. Biomass and Bioenergy 26(4):305-317.

Uhl, C.; R. Buschbacher y A.S. Serro E. 1988. Abandoned pastures in eastern Amazonia. I. Pattern of plant succession. J. Ecol. 76:663-681.

Young, H.F. y T.C. Tryon. 1978. A national forest biomass inventory. IUFRO, S 4.01 Forest inventory meeting in Bucharest Romania. 11 p.

Wenger, K.F. 1984. Forestry Handbook. Society of American Foresters. A. Wiley \& Sons. $1335 p$.

Whittaker, R.H. y P.L. Marks. 1975. Methods of assessing terrestrial productivity. In: Leith, H. y R.H. Wittaker. (eds), Primary productivity of the Biosphere. Springer-Nueva York. pp: 55-118.» 